\title{
ANÁLISE DE INVESTIMENTO EM PLATAFORMAS COLHEDORAS DE MILHO EM ESPAÇAMENTO REDUZIDO: EFEITO DE OSCILAÇÕES DA PRODUTIVIDADE, DO PREÇO DO MILHO E DA SEMENTE
}

\author{
VILNEI DE O. DIAS ${ }^{1}$, DICEU J. FERRI' ${ }^{2}$, AIRTON DOS S. ALONÇO ${ }^{3}$, \\ RENATO S. DE SOUZA ${ }^{4}$
}

RESUMO: O objetivo deste trabalho foi avaliar a viabilidade econômica do investimento em plataformas colhedoras de milho em espaçamento reduzido quanto a aumentos percentuais de produtividade, do preço do milho e da semente. Para tal, foi confeccionado fluxo de caixa diferencial e, a partir desse, determinaram-se a Taxa Interna de Retorno (TIR) e a Razão Benefício/Custo (B/C) do investimento para as combinações de aumentos de produtividade em diferentes níveis tecnológicos. Fez-se análise de sensibilidade para variações no preço pago pelo produto e nas despesas com sementes para o indicador Valor Presente Líquido (VPL). Foram consideradas áreas de 100 a 300 ha e produtividades de 70; 100; 130 e $160 \mathrm{sc} \mathrm{ha}^{-1}$. Concluiu-se que os acréscimos de produtividade afetam positivamente a TIR e a razão B/C. Quando esses acréscimos são superiores a 7,5\%, o investimento é viável economicamente na maioria das situações analisadas. Variações no preço de sementes têm baixo efeito sobre o investimento, e oscilações no preço do milho são toleradas na maioria das situações testadas.

PALAVRAS-CHAVE: engenharia agrícola, análise de sensibilidade, Zea mays.

\section{CORN GATHERER PLATFORM INVESTMENT ANALYSIS IN REDUCED SPACING: OSCILLATION EFFECT IN THE PRODUCTIVITY, CORN AND SEED PRICE}

\begin{abstract}
The objective of this work was to evaluate the economical viability of the investment in corn gatherer platforms in reduced spacing for the productivity percentile increases of the seed and corn price. For such purpose, a differential cash flow was accomplished and from this same cash it was determined the Return Internal Tax (RIT) and investment Benefit/Cost Rate (B/C) for the productivity increasing combinations in different technological levels. It was accomplished a sensibility analysis for variations in the product paid price and in the expenses with seeds for the Liquid Present Value (LPV) indicator. Areas from 100 to 300 ha were considered and productivities of 70;100; 130 and $160 \mathrm{sc} \mathrm{ha}^{-1}$. It was concluded that the productivity increments affect RIT and $\mathrm{B} / \mathrm{C}$ rate. Whenever these increments are superior to $7.5 \%$, the investment is economically viable in most of the analyzed situations. Price variations of seeds have low effect on the investment; corn price oscillations are tolerated in most of the tested situations.
\end{abstract}

KEYWORDS: agricultural engineering, sensibility analysis, Zea mays.

\section{INTRODUÇÃO}

O milho (Zea mays L.), em função do seu potencial produtivo, composição química e valor nutritivo, constitui-se em um dos mais importantes cereais cultivados e consumidos no mundo (FANCELLI \& DOURADO NETO, 2000). De acordo com a CONAB (2007), a produtividade média brasileira na safra 2005/2006 foi de 54,66 sc ha ${ }^{-1}$, e em alguns Estados brasileiros foi de $13,33 \mathrm{sc} \mathrm{ha}^{-1}$. Em virtude das modificações mais recentes introduzidas nos genótipos de milho, tais como menor estatura de planta e altura de inserção de espiga, menor esterilidade de plantas, menor

\footnotetext{
${ }^{1}$ Eng $^{\mathrm{o}}$ Agrônomo, Doutorando em Engenharia Agrícola, PPGEA/UFSM, Santa Maria - RS, Fone: (0XX55) 9111.1215, vilnei@ymail.com.

${ }^{2}$ Eng $^{\mathrm{o}}$ Agrônomo, Santa Maria - RS.

${ }^{3}$ Eng ${ }^{\text {o }}$ Agrícola, Doutor, Prof. Adjunto, Departamento de Engenharia Rural, UFSM, Santa Maria - RS.

${ }^{4}$ Eng $^{\mathrm{o}}$ Agrônomo, Doutor, Prof. Adjunto, Departamento de Educação Agrícola e Extensão Rural, UFSM, Santa Maria - RS.

Recebido pelo Conselho Editorial em: 21-11-2007

Aprovado pelo Conselho Editorial em: 26-4-2009
} 
duração do subperíodo pendoamento-espigamento, plantas com folhas de angulação mais ereta e elevado potencial produtivo, torna-se necessário reavaliar as recomendações de práticas de manejo para essa cultura (SANGÓI et al., 2006).

Uma prática que vem sendo testada em inúmeras pesquisas é a redução do espaçamento entre linhas de cultivo (REUNIÃO..., 2005). Os aumentos de produtividade advindos do emprego dessa tecnologia são bastante variáveis de acordo com diversos fatores, ocasionando imprecisão quanto ao aumento real que pode acontecer. Quando o agricultor decide semear milho em espaçamento reduzido, deve prever a aquisição de plataforma de colheita capaz de coletar plantas em espaçamentos menores, o que requer investimentos elevados em curto prazo.

DIAS et al. (2007) estudaram a viabilidade econômica de tal investimento e constataram que esse é viável economicamente em lavouras com práticas de manejo que visam a potencializar o rendimento da cultura, relatando que a viabilidade do investimento depende basicamente de três variáveis: escala de produção, produtividade da lavoura e ganhos de produtividade com a adoção da técnica, que estão relacionados ao nível de manejo empregado pelo produtor. Os autores estudaram o efeito de variações de acréscimos de produtividade sobre o indicador Valor Presente Líquido (VPL) e constataram que aumentos de $6 \%$ tornam o investimento viável do ponto de vista do VPL na maioria das situações estudadas, exceto para áreas inferiores a 100 ha associadas a produtividades inferiores a $65 \mathrm{sc} \mathrm{ha}^{-1}$.

Além da produtividade, outros elementos que afetam a viabilidade econômica são os preços dos insumos e o preço de venda do produto (PONCIANO et al., 2004). Os riscos de mercado, associados à volatilidade dos preços, devem ser considerados na análise de investimento, pois estão fora do controle do produtor e podem influenciar significativamente na rentabilidade da atividade. Dessa forma, maior atenção deve ser dada a tal fator, visto que a estacionalidade da produção e a sazonalidade dos preços são fenômenos associados à cultura do milho. Para elucidar os efeitos de flutuações no mercado, deve-se recorrer à técnica de análise de sensibilidade. Para BUARQUE (1991), a análise de sensibilidade consiste em medir em que magnitude uma alteração prefixada em uma ou mais variáveis do projeto altera o resultado final. Assim, o objetivo deste trabalho foi verificar a viabilidade econômica de investimento em plataformas colhedoras de milho, em espaçamento reduzido, quanto ao efeito de oscilações no preço do milho, da semente e da produtividade.

\section{MATERIAL E MÉTODOS}

Este estudo é complementar aos de ALONÇO et al. (2006) e DIAS et al. (2006a, b; 2007), sendo que maiores detalhes metodológicos podem ser obtidos nessas publicações. O trabalho foi desenvolvido na Universidade Federal de Santa Maria (UFSM), Núcleo de Ensaio de Máquinas Agrícolas (NEMA), em cooperação com o Núcleo de Estudos e Pesquisas em Economia Agroindustrial (NEPEA).

Os dados referentes às plataformas de colheita para milho, em espaçamento reduzido, foram obtidos junto a três revendedores de plataformas, representando as marcas mais comercializadas no Estado do Rio Grande do Sul, sendo coletados preços e especificações técnicas dos diferentes modelos disponíveis de tais marcas.

Para a avaliação do efeito do aumento da produtividade na viabilidade da aquisição de plataformas para colheita de milho, em espaçamento reduzido, os acréscimos de produtividade testados foram de 2,5; 5,0; 7,5 e 10\% sobre quatro produtividades: 70; 100; 130 e $160 \mathrm{sc} \mathrm{ha}^{-1}$. Esses percentuais foram aplicados sobre as produtividades consideradas, sendo o valor obtido multiplicado pelo preço real (deflacionado) médio da saca de $60 \mathrm{~kg}$ de milho, no período de janeiro de 2001 a dezembro de 2005, cujo valor calculado foi de $\mathrm{R} \$ 20,00$. Os dados da série temporal de preços nominais foram obtidos junto à EMATER-RS. Para a obtenção das entradas do fluxo de caixa, esse valor foi somado ao valor residual da plataforma, fixado em $15 \%$ do valor novo. Na averiguação do efeito de oscilações nos preços da semente e do milho sobre a viabilidade da 
aquisição da plataforma, realizou-se revisão bibliográfica para obter-se o aumento médio de produtividade com a adoção do espaçamento reduzido, em condições experimentais, sendo que a média encontrada foi de 5\%. Esse percentual foi aplicado sobre as produtividades consideradas e o valor obtido multiplicado pelo preço considerado da saca de $60 \mathrm{~kg}$ de milho. A partir da elaboração do fluxo de caixa diferencial com horizonte de 10 anos, calcularam-se os indicadores VPL, TIR e Relação Benefício/Custo.

As saídas do fluxo de caixa constaram de despesas de investimento e despesas operacionais; as primeiras consideram o preço inicial da plataforma pago à vista. As despesas operacionais consideradas foram manutenção da plataforma e gasto adicional com sementes, devido às recomendações de possível elevação da densidade de plantas em condições de espaçamento reduzido. $\mathrm{O}$ aumento considerado foi de $10 \mathrm{mil}_{\text {plantas }} \mathrm{ha}^{-1}$ ao preço médio de $\mathrm{R} \$ 105,00$ por saca de 60 mil sementes. As plataformas foram dimensionadas, segundo MIALHE (1974), para áreas de $100 ; 150 ; 200 ; 250$ e 300 ha. Para o dimensionamento, consideraram-se jornada de trabalho de $10 \mathrm{~h}$, período de colheita do milho inferior a 21 dias, com a finalidade de evitar perdas na lavoura, velocidade de $5,0 \mathrm{~km} \mathrm{~h}^{-1}$ e eficiência de campo de $75 \%$. Para a averiguação da influência do aumento da produtividade com adoção da técnica, foram utilizados os indicadores de viabilidade econômica Taxa Interna de Retorno (TIR) e razão Benefício/Custo (B/C), calculados com base em fluxo de caixa diferencial de 10 anos.

Baseado no fluxo de caixa, foi feita análise de sensibilidade para despesas com sementes e preço pago pela saca de $60 \mathrm{~kg}$ de milho. Para a análise de sensibilidade, foi usado o indicador Valor Presente Líquido (VPL). O VPL pode ser definido como a soma algébrica dos valores descontados do fluxo de caixa a ele associado. Em outras palavras, é a diferença do valor presente das entradas menos o valor presente das saídas do fluxo de caixa de um projeto de investimento (SILVA \& FONTES, 2005); ou, ainda, corresponde à concentração de todos os valores de um fluxo de caixa descontados para a data zero ou presente (KREUZ et al., 2005). Segundo HIRSCHFELD(1982), o VPL é dado pela eq.(1):

$$
\mathrm{VPL}=-\mathrm{I}+\sum \frac{\mathrm{FLt}}{(1+\mathrm{K})^{\mathrm{t}}}
$$

em que,

VPL - valor presente líquido;

I - investimento de capital na data zero;

FLt - líquido no período $t$ do fluxo de caixa, e

$\mathrm{k}$ - taxa mínima de atratividade (TMA) para realizar o investimento, ou custo de oportunidade do capital fixada em $8 \%$ a.a.

Por definição, a TIR de um projeto é a taxa de desconto que torna nulo o VPL do fluxo de caixa do investimento. É aquela que torna o valor presente dos lucros futuros equivalentes aos dos gastos realizados com o projeto, caracterizando, assim, a taxa de remuneração do capital investido (PONCIANO et al., 2004). Logo, a TIR é obtida, segundo PONCIANO et al. (2004) por:

$$
\mathrm{O}=-\mathrm{I}+\sum \frac{\mathrm{FLt}}{(1+\mathrm{TIR})^{\mathrm{t}}}
$$

\section{RESULTADOS E DISCUSSÃO}

$\mathrm{Na}$ Tabela 1, mostram-se os resultados referentes à influência de acréscimos percentuais de produtividade (AP\%) sobre a Taxa Interna de Retorno (TIR\%) em quatro níveis tecnológicos. Observa-se que, para a menor produtividade, aumentos de produtividade de $2,5 \%$ não resultaram em TIRs positivas. Esse fato torna a se repetir na produtividade de $100 \mathrm{sc} \mathrm{ha}^{-1}$, mas somente para áreas de até 150 ha e no terceiro nível de manejo $\left(130 \mathrm{sc} \mathrm{ha}^{-1}\right)$ em áreas inferiores a 100 ha. Para o nível de maior produtividade, $160 \mathrm{sc} \mathrm{ha}^{-1}$, para quase todas as situações, o investimento é viável pelo indicador TIR pelo fato de esse ser superior ao custo de oportunidade do capital. A exceção 
deve-se à situação de área de 100 ha e variação de produtividade de 2,5\%, em que o valor da TIR de 3,20 é inferior a custo de oportunidade do capital considerado.

A TIR indica a viabilidade de um investimento, comparada a TMA, considerada, nesse caso, como o custo de oportunidade do capital, igual a $8 \%$. Analisando-se a menor produtividade, e se considerarmos a TMA de $8 \%$ ao ano, o investimento será viável na área de 100 ha apenas para acréscimos de 7,5\%, o que necessitaria de condições ótimas de cultivo para ser obtido. O produtor que tem nível de produtividade baixo como esse, se apenas optar por reduzir o espaçamento entre linhas, pode ter insucesso, visto que tal prática nos dá maiores retornos que a TMA em altas produtividades e/ou em maiores áreas. No segundo nível tecnológico avaliado, aumentos de 2,5\% na produtividade já tornam a TIR maior que a TMA em áreas superiores a 300 ha, e aumentos de $5 \%$ tornam o investimento viável desde 100 ha. Quando a produtividade considerada é de $130 \mathrm{sc} \mathrm{ha}^{-1}$, acréscimos de 2,5\% tornam o investimento atrativo em áreas de dimensões superiores a 200 ha. Isto indica que, em produtividades superiores, mesmo com pequenos acréscimos, a adoção do espaçamento reduzido pode ser atrativa financeiramente.

Verificou-se, também, que, em cultivos que atingem produtividades de $160 \mathrm{sc} \mathrm{ha}^{-1}$, aumentos superiores a 2,5\% tornam o investimento atrativo para áreas iguais a $150 \mathrm{ha}$, e aumentos superiores a 5\% mostram que o investimento nessa técnica é viável para qualquer área igual ou superior a 100 ha. A afirmação anterior confere com os resultados de ALONÇO et al. (2006), que afirmam que, em elevadas produtividades, a técnica de redução do espaçamento entre linhas pode ser economicamente atrativa. Quando os acréscimos considerados são de 7,5 e 10\%, pode-se ter TIRs bastante altas, indicando maior viabilidade econômica nessas situações. DIAS et al. (2007) estudaram a TIR desse investimento, porém com aumento fixo de 5\% sobre quatro produtividades. A TIR máxima encontrada pelos autores foi de 55,04\% associada à produtividade de $140 \mathrm{sc} \mathrm{ha}^{-1} \mathrm{e}$ áreas superiores a 300 ha, sendo que acréscimos em área não ocasionaram aumentos significativos na TIR.

TABELA 1. Influência de acréscimos percentuais de produtividade (AP\%) sobre a Taxa Interna de Retorno (TIR\%) em três níveis tecnológicos. Productivity percentage increase influence (AP\%) on the Internal Rate of Return (TIR\%) in three technological levels.

\begin{tabular}{|c|c|c|c|c|c|}
\hline & \multicolumn{5}{|c|}{ Área (ha) } \\
\hline & 100 & 150 & 200 & 250 & 300 \\
\hline $\mathrm{AP}(\%)$ & \multicolumn{5}{|c|}{ 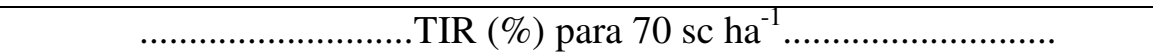 } \\
\hline 2,5 & - & - & - & - & - \\
\hline 5,0 & - & 7,20 & 13,00 & 18,40 & 23,60 \\
\hline 7,5 & 9,20 & 18,40 & 26,90 & 34,90 & 42,60 \\
\hline \multirow[t]{2}{*}{10,0} & 16,70 & 28,50 & 39,60 & 50,20 & 60,60 \\
\hline & \multicolumn{5}{|c|}{ 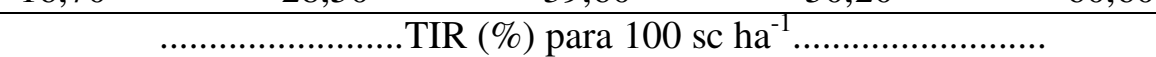 } \\
\hline 2,5 & - & - & 3,90 & 7,70 & 11,40 \\
\hline 5,0 & 8,00 & 16,90 & 25,00 & 32,60 & 40,00 \\
\hline 7,5 & 18,70 & 31,30 & 43,10 & 54,50 & 65,70 \\
\hline \multirow[t]{2}{*}{10,0} & 28,30 & 44,60 & 60,20 & 75,60 & 91,00 \\
\hline & \multicolumn{5}{|c|}{ 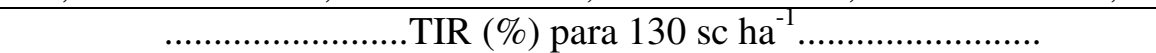 } \\
\hline 2,5 & - & 5,40 & 10,80 & 15,90 & 20,60 \\
\hline 5,0 & 14,60 & 25,70 & 36,00 & 45,90 & 55,60 \\
\hline 7,5 & 27,30 & 43,30 & 58,50 & 73,50 & 88,30 \\
\hline \multirow[t]{2}{*}{10,0} & 39,10 & 60,00 & 80,40 & 100,60 & 120,70 \\
\hline & \multicolumn{5}{|c|}{ 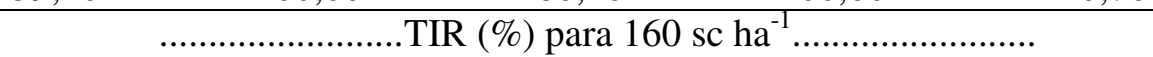 } \\
\hline 2,5 & 3,20 & 10,55 & 17,15 & 23,30 & 29,20 \\
\hline 5,0 & 20,65 & 34,00 & 46,55 & 58,75 & 70,75 \\
\hline 7,5 & 35,60 & 54,90 & 73,70 & 92,30 & 110,75 \\
\hline 10,0 & 49,60 & 75,15 & 100,40 & 125,50 & 150,60 \\
\hline
\end{tabular}


Na Tabela 2, apresenta-se a Razão Benefício/Custo do investimento em relação aos aumentos de produtividade percentuais e tamanhos de área estudados. Pode-se perceber que, para a menor produtividade $\left(70 \mathrm{sc} \mathrm{ha}^{-1}\right)$, esse índice nos mostra que a viabilidade será atingida em áreas de 100 ha e com aumentos de produtividade de 7,5\%. Já para o nível tecnológico, que atinge produtividade de $100 \mathrm{sc} \mathrm{ha}^{-1}$, esse investimento torna-se viável com aumentos de 5\% para área de 100 ha. Para áreas superiores a 200 ha, que alcançam produtividades de $130 \mathrm{sc} \mathrm{ha}^{-1}$, o benefício torna-se maior que o custo, com acréscimos na produtividade de $2,5 \%$.

Quando foram consideradas situações cuja produtividade atingida foi de $160 \mathrm{sc} \mathrm{ha}^{-1}$, somente áreas de 100 ha com aumento na produtividade de apenas 2,5\% tornaram o investimento economicamente inviável. Esses resultados demonstram que, com maiores níveis de manejo, altas produtividades e em áreas relativamente maiores, a aquisição de plataformas colhedoras para espaçamento reduzido torna-se economicamente viável também pelo indicador Razão Benefício/Custo, mesmo com pequenos acréscimos na produtividade, confirmando o que DIAS et al. $(2006 \mathrm{a}, \mathrm{b} ; 2007)$ encontraram em trabalhos anteriores.

TABELA 2. Influência de acréscimos percentuais de produtividade (APP, \%) sobre a relação Benefício/Custo em três níveis tecnológicos. Productivity percentage increase influence (APP,\%) on the benefit / cost relation in three technological levels.

\begin{tabular}{|c|c|c|c|c|c|}
\hline & \multicolumn{5}{|c|}{ Área (ha) } \\
\hline & 100 & 150 & 200 & 250 & 300 \\
\hline $\operatorname{APP}(\%)$ & \multicolumn{5}{|c|}{$\mathrm{B} / \mathrm{C}$ para $70 \mathrm{sc} \mathrm{ha}^{-1}$} \\
\hline 2,5 & 0,40 & 0,55 & 0,65 & 0,75 & 0,85 \\
\hline 5,0 & 0,80 & 1,05 & 1,30 & 1,50 & 1,65 \\
\hline 7,5 & 1,15 & 1,55 & 1,90 & 2,20 & 2,45 \\
\hline 10,0 & 1,50 & 2,05 & 2,50 & 2,90 & 3,25 \\
\hline \multicolumn{6}{|c|}{$\mathrm{B} / \mathrm{C}$ para $100 \mathrm{sc} \mathrm{ha}^{-1}$} \\
\hline 2,5 & 0,55 & 0,75 & 0,95 & 1,05 & 1,20 \\
\hline 5,0 & 1,10 & 1,45 & 1,80 & 2,10 & 2,35 \\
\hline 7,5 & 1,60 & 2,15 & 2,65 & 3,10 & 3,50 \\
\hline 10,0 & 2,10 & 2,90 & 3,55 & 4,15 & 4,65 \\
\hline \multicolumn{6}{|c|}{$\mathrm{B} / \mathrm{C}$ para $130 \mathrm{sc} \mathrm{ha}^{-1}$} \\
\hline 2,5 & 0,70 & 0,95 & 1,20 & 1,40 & 1,55 \\
\hline 5,0 & 1,40 & 1,90 & 2,35 & 2,70 & 3,05 \\
\hline 7,5 & 2,05 & 2,80 & 3,45 & 4,05 & 4,50 \\
\hline 10,0 & 2,70 & 3,70 & 4,60 & 5,35 & 6,00 \\
\hline \multicolumn{6}{|c|}{$\mathrm{B} / \mathrm{C}$ para $160 \mathrm{sc} \mathrm{ha}^{-1}$} \\
\hline 2,5 & 0,90 & 1,20 & 1,45 & 1,70 & 1,90 \\
\hline 5,0 & 1,70 & 2,30 & 2,85 & 3,30 & 3,70 \\
\hline 7,5 & 2,50 & 3,45 & 4,25 & 4,95 & 5,55 \\
\hline 10,0 & 3,30 & 4,55 & 5,65 & 6,60 & 7,40 \\
\hline
\end{tabular}

Na Tabela 3, estão os valores da análise de sensibilidade para variações no preço da saca de 60 mil sementes sobre o indicador Valor Presente Líquido (VPL). Os valores em reais indicam quanto deve custar a saca de semente para que o investimento se torne viável. Os valores relativos têm como base o preço médio de $\mathrm{R} \$ 105,00$. Verifica-se que, para as propriedades com 100 ha, a inviabilidade do investimento está associada a baixas produtividades, como apresentado para o nível mais baixo de manejo ( $70 \mathrm{sc} \mathrm{ha}^{-1}$ ), em que a semente deve ser $91 \%$ mais barata para que o investimento se torne viável; e no caso em que a produtividade é de $100 \mathrm{sc} \mathrm{ha}^{-1}$, para o qual pequenas oscilações $(0,5 \%)$ no preço da semente podem indicar inviabilidade para o investimento. Deve-se salientar que tal percentual é em relação às sementes adicionais necessárias, em caso de elevação da densidade de plantas. 
TABELA 3. Análise de sensibilidade para variações no preço da saca de 60 mil sementes sobre o indicador Valor Presente Líquido (VPL) em quatro níveis de produtividade. Sensitivity Analysis for changes in the bag price of 60 thousand seeds on the Net Present Value indicator (NPV) in four productivity levels.

\begin{tabular}{|c|c|c|c|c|c|c|c|c|c|c|}
\hline & \multicolumn{10}{|c|}{ Área (ha) } \\
\hline & \multicolumn{2}{|c|}{100} & \multicolumn{2}{|c|}{150} & \multicolumn{2}{|c|}{200} & \multicolumn{2}{|c|}{250} & \multicolumn{2}{|c|}{300} \\
\hline & $\mathrm{R} \$ *$ & $\% * *$ & $\mathrm{R} \$$ & $\%$ & $\mathrm{R} \$$ & $\%$ & $\mathrm{R} \$$ & $\%$ & $\mathrm{R} \$$ & $\%$ \\
\hline 70 & $-74,55$ & $-91,0$ & 90,30 & $-14,0$ & 172,70 & 64,5 & 222,20 & 111,6 & 255,20 & 143,0 \\
\hline 100 & 105,50 & 0,5 & 270,30 & 157,4 & 352,70 & 235,9 & 402,50 & 283,3 & 435,60 & 314,9 \\
\hline 130 & 285,50 & 171,9 & 450,40 & 329,0 & 532,70 & 407,3 & 582,20 & 454,5 & 615,30 & 486,0 \\
\hline 160 & 465,50 & 433,3 & 630,30 & 600,3 & 712,70 & 678,8 & 762,20 & 725,9 & 795,15 & 757,3 \\
\hline
\end{tabular}

*Valor em reais que anula o VPL; **Preço médio de referência: $\mathrm{R} \$ 105,00$.

Áreas de 150 ha podem ser comparadas àquelas de 100 ha, em que o menor nível de manejo exige decréscimo no preço da saca, porém da ordem de $14 \%$. A diferença está que, nas áreas de $150 \mathrm{ha}$, a viabilidade aparece a partir de produtividade de $100 \mathrm{sc} \mathrm{ha}^{-1}$, na qual o preço da saca de sementes pode aumentar em torno de $150 \%$ que a produção de milho no espaçamento reduzido irá cobrir o investimento da plataforma.

Nas maiores áreas estudadas (200; 250 e 300 ha), mesmo nas condições de manejo menos favoráveis, com produtividade de 70 sacas $^{-1} \mathrm{a}^{-1}$, a viabilidade do investimento é menos suscetível à elevação do preço da semente. Para essas mesmas áreas, a situação de viabilidade é constituída em qualquer outro nível tecnológico estudado, podendo o valor da saca ultrapassar $64 \%$, conforme dados da Tabela 3. DIAS et al. (2007) concluíram que a faixa que necessitava de estudos mais profundos da viabilidade econômica do investimento, era inferior a 300 ha, o que também foi observado no presente trabalho.

Na Tabela 4, encontram-se os resultados da análise de sensibilidade para preço da saca de $60 \mathrm{~kg}$ de milho sobre o indicador Valor Presente Líquido (VPL). Da mesma forma apresentada na Tabela 3, os valores absolutos, em reais, indicam quanto poderia atingir o valor da saca de milho para que o investimento aponte viabilidade econômica. Os valores percentuais são relativos ao preço médio deflacionado do período que compreende janeiro de 2001 a dezembro de 2005, que é de $\mathrm{R} \$ 20,00$.

Para a produtividade de $70 \mathrm{sc} \mathrm{ha}^{-1}$, o milho deveria valer mais do que a média do período considerado para que o investimento se torne viável para as áreas de 100 e 150 ha. Quando estudada a área de $100 \mathrm{ha}$, ficou demonstrado que somente nas produtividades de $130 \mathrm{e} 160 \mathrm{sc} \mathrm{ha}^{-1} \mathrm{o}$ investimento aceitará maiores variações negativas no preço pago pela saca de milho. Para áreas de 150 ha, com produtividades iguais ou superiores a $100 \mathrm{sc} \mathrm{ha}^{-1}$, já são aceitas quedas no preço do milho, mas de apenas $27,6 \%$ nessa mesma produtividade.

SANGÓI et al. (2006) estudaram níveis de manejo para a cultura do milho e verificaram que o maior investimento em práticas de manejo e insumos incrementa o rendimento de grãos e a margem bruta da cultura, independentemente do tipo de base genética, isto é, a cultura do milho pode ser mais rentável economicamente em situações de elevado nível tecnológico, devido ao seu potencial produtivo (DIAS et al., 2007). Este trabalho vem a ratificar as conclusões dos autores, pois, do ponto de vista desse investimento, maiores níveis produtivos dão maior garantia de viabilidade econômica do investimento.

Nas áreas estudadas de 200; 250 e 300 ha, a viabilidade torna-se concreta desde a menor produtividade avaliada, mesmo que nessa o preço da saca do milho sofra redução de $16,1 \%$. Analisando a área máxima (300 ha), constata-se que, mesmo no menor nível produtivo $\left(70 \mathrm{sc} \mathrm{ha}^{-1}\right)$, o investimento tolera quedas de até $36 \%$ para se tornar inviável, e no maior $\left(160 \mathrm{sc} \mathrm{ha}^{-1}\right)$, tolera decréscimos de até $71,90 \%$. 
TABELA 4. Análise de sensibilidade para preço da saca de $60 \mathrm{~kg}$ de milho sobre o indicador Valor Presente Líquido (VPL) em quatro níveis de produtividade. Sensitivity Analysis for the bag price of $60 \mathrm{~kg}$ of maize on the Net Present Value indicator (NPV) in four productivity levels.

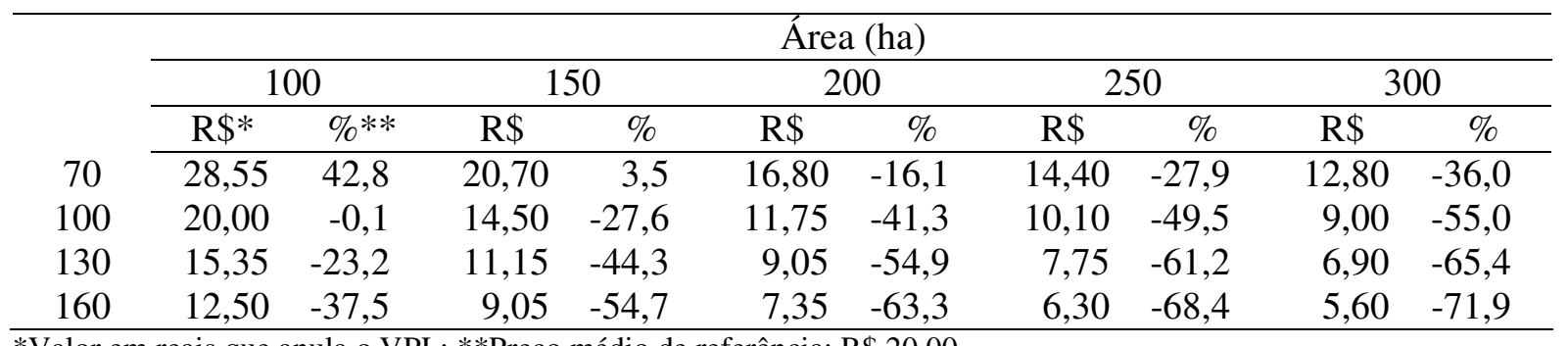

*Valor em reais que anula o VPL; **Preço médio de referência: $\mathrm{R} \$ 20,00$.

Durante o período considerado, tomando-se as médias anuais, o menor preço médio ocorreu em 2001, quando atingiu R\$16,24. No mesmo ano, o menor preço mensal ocorreu no mês de abril, quando o produto estava cotado a $\mathrm{R} \$ 12,83$. Isso indica que o investimento pode ser seguro nas quatro produtividades testadas com relação a quedas no preço do milho, especialmente em áreas acima de 200 ha.

\section{CONCLUSÕES}

Nas condições de realização anteriormente descritas neste estudo, pode-se concluir que o manejo é o diferencial na viabilidade econômica do investimento em plataformas de colheita de milho em espaçamento reduzido, pois, como demonstrado, em áreas com aplicação de melhor manejo, conseguem-se maiores produtividades e, consequentemente, maior receita por área; pequenos acréscimos de produtividade viabilizam o uso da técnica.

O preço da semente é menos importante que o preço da saca de milho na maioria das situações estudadas, o que se deve ao fato de a saca de milho corresponder a uma parcela diferencial na receita proporcionalmente maior do que a semente no custo.

\section{REFERÊNCIAS}

ALONÇO, A. dos S.; DIAS, V. de O.; SOUZA, R.S. de; MEDEIROS, F.A.; MASSOCO, D. Viabilidade econômica da aquisição de plataforma de colheita para milho em espaçamento reduzido. In: CONGRESSO BRASILEIRA DE ENGENHARIA AGRÍCOLA, 36., 2006, João Pessoa. Anais... Jaboticabal: Associação Brasileira de Engenharia Agrícola, 2006. 1 CD-ROM.

BUARQUE, C. Avaliação econômica de projetos. 6. ed. Rio de Janeiro: Campus, 1991. 266 p.

CONAB. Central de Informações Agropecuárias. Disponível em: <http://

http://www.conab.gov.br/conabweb/index.php?PAG=131>. Acesso em: 31 out. 2007.

DIAS, V. de O.; ALONÇO, A. dos S.; SOUZA, R.S. de. Análise da viabilidade econômica do investimento em plataformas de colhedoras de milho em espaçamento reduzido. Engenharia Agrícola, Jaboticabal, v.27, n.2, p.463-470, 2007.

DIAS, V. de O.; ALONÇO, A. dos S.; SOUZA, R.S. de. Avaliação econômica do investimento em plataformas de colheita mecanizada de milho em espaçamento reduzido. In: JORNADA DE JOVENS INVESTIGADORES DA AUGM, 14., 2006, Campinas. Anais... Montevidéu: Associação das Universidades do Grupo de Montevidéu, 2006a. 1 CD-ROM.

DIAS, V. de O.; ALONÇO, A. dos S.; SOUZA, R.S. de. Viabilidade econômica da adoção de espaçamento reduzido na cultura do milho. In: ENCONTRO ANUAL DE INICIAÇÃO CIENTÍFICA, 15., 2006, Ponta Grossa. Anais... Ponta Grossa: Universidade Estadual de Ponta Grossa, 2006b. 1 CD-ROM. 
ESPERANCINI, M.S.T.; PAES, A. R. Análise de investimentos da produção de café nos sistemas irrigado e convencional, na região de Botucatu, Estado de São Paulo. Informações Econômicas, São Paulo, v.35, n.4, 2005.

FANCELLI, A.L.; DOURADO NETO, D. Produção de milho. Guaiba: Agropecuária, 2000. 360 p. HIRSCHFELD, H. Engenharia econômica. São Paulo: Atlas, 1982. 334 p.

KREUZ, SOUZA, A.; SCHUCK, E.; PETRI, J. L. Avaliação econômica de alternativas de investimento no agronegócio da uva no meio oeste catarinense. Revista Brasileira de Fruticultura, Jaboticabal, v. 27, n.2, p. 230-237, 2005.

MIALHE, L. G. Manual de mecanização agrícola. 11. ed. São Paulo: Ceres, 1974. 301p.

PONCIANO, N.J.; SOUZA, P.M. de; MATA, H.T. de C.; VIEIRA, J.R.; MORGADO, I.F. Análise de viabilidade econômica e de risco da fruticultura na região Norte Fluminense. Revista de Economia Rural, Rio de Janeiro, v.42, n.4, p.615-635, 2004.

REUNIÃO TÉCNICA ANUAL DE PESQUISA DE MILHO E SORGO. Indicações técnicas para cultivo de milho e sorgo no Rio Grande do Sul 2005/2006. Porto Alegre: FEPAGRO/EmaterRS/ASCAR, 2005. $155 \mathrm{p}$.

SANGÓI, L.; ERNANI, P.R.; SILVA, P.R.F. da; HORN, D.; SCHMITT, A.; SCHWEITZER, C.; MOTTER, F. Rendimento de grãos e margem bruta de cultivares de milho com variabilidade genética contrastante em diferentes sistemas de manejo. Ciência Rural, Santa Maria, v.36, n.3, p.747-755, 2006.

SILVA, M.L. da; FONTES, A.A. Discussão sobre os critérios de avaliação econômica: valor presente líquido (VPL), valor anual equivalente (VAE) e valor esperado da terra (VET). Revista Árvore, Viçosa, v.29, n.6, p.931-936, 2005. 